\title{
Affine Poisson Groups and WZW Model ${ }^{\star}$
}

\author{
Ctirad KLIMČÍK
}

Institute de mathématiques de Luminy, 163, Avenue de Luminy, 13288 Marseille, France

E-mail: klimcik@iml.univ-mrs.fr

Received October 31, 2007; Published online January 11, 2008

Original article is available at http://www.emis.de/journals/SIGMA/2008/003/

\begin{abstract}
We give a detailed description of a dynamical system which enjoys a PoissonLie symmetry with two non-isomorphic dual groups. The system is obtained by taking the $q \rightarrow \infty$ limit of the $q$-deformed WZW model and the understanding of its symmetry structure results in uncovering an interesting duality of its exchange relations.
\end{abstract}

Key words: Poisson-Lie symmetry; WZW model

2000 Mathematics Subject Classification: 81T40

\section{Introduction}

Poisson-Lie symmetry of a dynamical system is a generalization of the standard concept of the Hamiltonian symmetry. Poisson-Lie symmetric dynamical system possess a distinguished subalgebra of observables which is isomorphic to the Poisson algebra of functions on the socalled affine Poisson group. Given an affine Poisson group, it is not difficult to construct a Poisson-Lie symmetric dynamical system, by using the theory of Heisenberg doubles or, in other terminology, symplectic grupoids [8]. However, it is certainly more interesting to discover a Poisson-Lie symmetry in a model which was proposed or studied for reasons independent from the symmetry considerations. This is what exactly happened when we have studied a $\varepsilon \rightarrow \infty$ limit of the $\varepsilon$-deformed WZW model. The Poisson-Lie symmetry, which we have discovered in the limit, appears in turn to be a very useful structure for the quantitative analysis of the model. In particular, we shall see that it permits to identify a remarkable duality of the exchange relations of the theory.

This paper is a short review of the original research article [6] and it is written with the goal to streamline the presentation by omitting various technical details. Its plan is as follows: first we give a compact introduction to the concepts of the affine Poisson group and of the Poisson-Lie symmetry and then we describe the Poisson-Lie symmetry of the $\varepsilon$-deformed WZW model for finite $\varepsilon$. In the subsequent Section 4, we identify the Poisson-Lie symmetry of the $\varepsilon \rightarrow \infty$ limit of the $\varepsilon$-deformed WZW model and, finally, the Section 5 will be devoted to the duality of the exchange relations.

\section{Poisson-Lie symmetry}

Let $\Pi_{B}$ denote a Poisson bivector on a Lie group manifold $B$. As it is well-known $[3,10,7,2]$, $\Pi_{B}$ gives rise to the Lie algebra structure on the space $\Omega^{1}(B)$ consisting of smooth 1 -form fields on $B$ :

$$
\{x d y, u d v\}_{\Omega(B)}=x u d\{y, v\}_{B}+x\{y, u\}_{B} d v+u\{x, v\}_{B} d y
$$

where $x, y, u, v$ are smooth functions on $B$ and $\{y, v\}_{B} \equiv \Pi_{B}(d y, d v)$.

${ }^{\star}$ This paper is a contribution to the Proceedings of the Seventh International Conference "Symmetry in Nonlinear Mathematical Physics" (June 24-30, 2007, Kyiv, Ukraine). The full collection is available at http://www.emis.de/journals/SIGMA/symmetry2007.html 
If, at the same time, the spaces of right and left invariant 1-form fields are respectively Lie subalgebras $\mathcal{G}_{L}$ and $\mathcal{G}_{R}$ of the Lie algebra $\left(\Omega^{1}(B),\{\cdot, \cdot\}_{\Omega}\right)$, we say that $B$ is equipped with an affine Poisson structure $\Pi_{B}[2]$.

A dynamical system is a triple $\left(P, \Pi_{P}, H\right)$, where $\Pi$ is a non-degenerate bivector field on a manifold $P$ and $H$ is a distinguished function on $P$ called the Hamiltonian.

Poisson-Lie symmetry of a dynamical system $\left(P, \Pi_{P}, H\right)$ is any smooth map $\mu$ from $\left(P, \Pi_{P}\right)$ into an affine Poisson group $\left(B, \Pi_{B}\right)$ such that:

1 ) the $\mu$-pull-backs of any $x, y \in \operatorname{Fun}(B)$ satisfy

$$
\left\{\mu^{*} x, \mu^{*} y\right\}_{P}=\mu^{*}\{x, y\}_{B}
$$

2) for any $x \in \operatorname{Fun}(B)$ it exists $y \in \operatorname{Fun}(B)$ fulfilling

$$
\left\{H, \mu^{*} x\right\}_{P}=\mu^{*} y .
$$

The map $\mu$ is often called a moment map and it induces at the same time the actions of both Lie algebras $\mathcal{G}_{L}$ and $\mathcal{G}_{R}$ on $P$. Indeed, to any $\lambda \in \mathcal{G}_{L}$ it corresponds the vector field $\Pi_{P}\left(\cdot, \mu^{*} \lambda\right)$ acting on functions on $P$ and to any $\rho \in \mathcal{G}_{R}$ it corresponds $\Pi_{P}\left(\cdot, \mu^{*} \rho\right)$. The Lie algebras $\mathcal{G}_{L}$ and $\mathcal{G}_{R}$ are then interpreted as the symmetry algebras of the dynamical system.

A Poisson-Lie symmetry is called equivariant if the affine Poisson bivector $\Pi_{B}$ vanishes at the unit element $e_{B}$ of the group $B$. If the symmetry is equivariant then Lie algebra $\mathcal{G}_{L}$ is necessarily isomorphic to the Lie algebra $\mathcal{G}_{R}$. However, even in this case the $\mathcal{G}_{L}$ action on $P$ need not to coincide with the $\mathcal{G}_{R}$ action. Non-equivariant symmetry is called anomalous. In the presence of the anomaly, $\mathcal{G}_{L}$ need not be even isomorphic to $\mathcal{G}_{R}$.

\section{$3 \quad \varepsilon$-deformed WZW model}

Let $G$ be a connected and simply connected simple compact Lie group, $G^{\mathbf{C}}$ its complexification and $L G^{\mathbf{C}}$ the group of loops, i.e. the group of smooth maps from a circle $S^{1}$ into $G^{\mathbf{C}}$. Let $B$ be a subgroup of $L G^{\mathbf{C}}$ containing only the loops which are boundary values of holomorphic maps $H(z)$ from the southern Riemann hemisphere into the complex group $G^{\mathbf{C}}$. Moreover, it is required that the value of the $H(z)$ at the south pole $z=0$ lies in the subgroup $A N$ of $G^{\mathbf{C}}$. (Remind that $A N$ is the subgroup of $G^{\mathbf{C}}$ determined by the Iwasawa decomposition $G^{\mathbf{C}}=G A N$.) Set

$$
L(\sigma) \equiv H\left(e^{i \sigma}\right)\left(H\left(e^{i \sigma}\right)\right)^{\dagger},
$$

where $\dagger$ stands for the standard Hermitian conjugation. In [11], Reshetikhin and Semenov-TianShansky have introduced the following affine Poisson structure on $B$ :

$$
\begin{aligned}
& \left\{L(\sigma) \stackrel{\otimes}{,} L\left(\sigma^{\prime}\right)\right\}_{B}=\left(L(\sigma) \otimes L\left(\sigma^{\prime}\right)\right) \varepsilon \hat{r}\left(\sigma-\sigma^{\prime}\right)-\left(1 \otimes L\left(\sigma^{\prime}\right)\right) \varepsilon \hat{r}\left(\sigma-\sigma^{\prime}+2 \pi i \epsilon\right)(L(\sigma) \otimes 1) \\
& \quad+\varepsilon \hat{r}\left(\sigma-\sigma^{\prime}\right)\left(L(\sigma) \otimes L\left(\sigma^{\prime}\right)\right)-(L(\sigma) \otimes 1) \varepsilon \hat{r}\left(\sigma-\sigma^{\prime}-2 \pi i \epsilon\right)\left(1 \otimes L\left(\sigma^{\prime}\right)\right) .
\end{aligned}
$$

Here $\varepsilon$ is the deformation parameter and

$$
\begin{aligned}
& \hat{r}\left(\sigma-\sigma^{\prime}\right)=r+C \operatorname{cotg} \frac{1}{2}\left(\sigma-\sigma^{\prime}\right), \\
& r=\sum_{\alpha>0} \frac{i|\alpha|^{2}}{2}\left(E^{-\alpha} \otimes E^{\alpha}-E^{\alpha} \otimes E^{-\alpha}\right), \\
& C=\sum_{\mu} H^{\mu} \otimes H^{\mu}+\sum_{\alpha>0} \frac{|\alpha|^{2}}{2}\left(E^{-\alpha} \otimes E^{\alpha}+E^{\alpha} \otimes E^{-\alpha}\right) .
\end{aligned}
$$

Our conventions for the normalisations of the standard step generators $E^{\alpha}$ can be found in [6]. 
Reshetikhin and Semenov-Tian-Shansky have interpreted the affine Poisson structure (1) as the $\varepsilon$-deformed current algebra since in the limit $\varepsilon \rightarrow 0$ it reduces to the standard Kac-Moody current algebra. It is well-known that the Kac-Moody algebra underlies the symmetry structure of the so-called chiral WZW model [1] which is a very important dynamical system from the point of view of the conformal field theory. Motivated by this fact, Lukyanov and Shatashvili [9] have formulated the following problem: Is there a dynamical system which would be Poisson-Lie symmetric with respect to the affine Poisson structure (1) and would reduce to the standard chiral WZW model in the limit $\varepsilon \rightarrow 0$ ? The affirmative answer to this question was given in [5] and it goes along the following lines:

The space Fun $(P)$ of the observables of the $\varepsilon$-deformed WZW model is Fun $(P)=$ Fun $(L G) \otimes$ $\operatorname{Fun}^{W}(T)$, where $\operatorname{Fun}^{W}(T)$ is the space of Weyl invariant functions on the Cartan torus $T \subset G$. The non-degenerate Poisson structure on $P$ is determined by the following Poisson brackets

$$
\begin{aligned}
& \{t \stackrel{\otimes}{,} k(\sigma)\}_{P}=(t \otimes k(\sigma))\left(H^{\mu} \otimes H^{\mu}\right), \quad\{t \stackrel{\otimes}{,} t\}_{P}=0, \\
& \left\{k(\sigma) \stackrel{\otimes}{,} k\left(\sigma^{\prime}\right)\right\}_{P}=\left(k(\sigma) \otimes k\left(\sigma^{\prime}\right)\right) \hat{r}_{\epsilon}\left(t, \sigma-\sigma^{\prime}\right)-\epsilon \hat{r}\left(\sigma-\sigma^{\prime}\right)\left(k(\sigma) \otimes k\left(\sigma^{\prime}\right)\right),
\end{aligned}
$$

where $\hat{r}_{\epsilon}(t, \sigma)$ is the well-known Felder elliptic dynamical $r$-matrix [4]:

$$
\hat{r}_{\epsilon}(t, \sigma)=\epsilon \rho\left(\frac{\sigma}{2 \pi}, i \epsilon\right) H^{\mu} \otimes H^{\mu}+\epsilon \sum_{\alpha} \frac{|\alpha|^{2}}{2} \sigma_{-\epsilon\langle\alpha, \ln t\rangle}\left(\frac{\sigma}{2 \pi}, i \epsilon\right) E^{\alpha} \otimes E^{-\alpha} .
$$

The moment map $\mu_{\epsilon}: P \rightarrow B$, that realizes the Poisson-Lie symmetry of the $\varepsilon$-deformed WZW model, is then given by a compact formula

$$
\mu_{\epsilon}(k(\sigma), t)=I w_{\epsilon}\left(k(\sigma) t^{-i \epsilon}\right) .
$$

Here the Iwasawa map $I w_{\epsilon}: L G^{\mathbf{C}} \rightarrow B$ is uniquely characterized by the property that for every $l(\sigma) \in L G^{\mathbf{C}}$ it exists $m(\sigma-i \epsilon) \in L G$ such that $l^{-1} I w_{\epsilon}(l)=m(\sigma)$.

In [5], it was proven the existence of a function $H_{\varepsilon}$ on the phase space $P$ which gives the standard chiral WZW Hamiltonian in the limit $\varepsilon \rightarrow 0$ and fulfils the condition 2) of the definition of the Poisson-Lie symmetry above. We take the function $H_{\varepsilon}$ as the Hamiltonian of the $\varepsilon$ deformed WZW model, knowing that its explicit form in variables $k(\sigma)$ and $t$ is not available.

\section{The limit $\varepsilon \rightarrow \infty$}

The $\varepsilon \rightarrow \infty$ limit of the $\varepsilon$-deformed current algebra (1) reads

$$
\begin{aligned}
\left\{L(\sigma) \stackrel{\otimes}{,} L\left(\sigma^{\prime}\right)\right\}_{\infty}= & \left(L(\sigma) \otimes L\left(\sigma^{\prime}\right)\right) \hat{r}\left(\sigma-\sigma^{\prime}\right)+\hat{r}\left(\sigma-\sigma^{\prime}\right)\left(L(\sigma) \otimes L\left(\sigma^{\prime}\right)\right) \\
& -(L(\sigma) \otimes 1)(r+i C)\left(1 \otimes L\left(\sigma^{\prime}\right)\right)-\left(1 \otimes L\left(\sigma^{\prime}\right)\right)(r-i C)(L(\sigma) \otimes 1) .
\end{aligned}
$$

It turns out that equation (3) defines an affine Poisson structure on the group $B$, which is qualitatively different from (1). Indeed, for finite $\varepsilon$, the Lie algebras $\mathcal{G}_{L}$ and $\mathcal{G}_{R}$ associated to the affine Poisson structure (1) turn out to be isomorphic to each other and also to Lie $(L G)$. However, in the limit $\varepsilon \rightarrow \infty, \mathcal{G}_{L}$ is still isomorphic to Lie $(L G)$ but $\mathcal{G}_{R}$ is isomorphic to the Lie algebra of another group which we denote as $\bar{B}$. In fact, $\bar{B}$ turns out to be the subgroup of $L G^{\mathbf{C}}$ containing only the loops which are boundary values of holomorphic maps $\bar{H}(z)$ from the northern Riemann hemisphere into the complex group $G^{\mathbf{C}}$. Moreover, it is required that the value of $\bar{H}$ at the north pole $z=\infty$ lies in the subgroup $G$ of $G^{\mathbf{C}}$.

The $\varepsilon \rightarrow \infty$ limit of the exchange relations (2) reads

$$
\begin{aligned}
& \left\{e^{a} \stackrel{\otimes}{,} k(\sigma)\right\}_{\infty}=-i\left(e^{a} \otimes k(\sigma)\right)\left(H^{\mu} \otimes H^{\mu}\right), \quad\{a \stackrel{\otimes}{,} a\}_{\infty}=0, \\
& \left\{k(\sigma) \stackrel{\otimes}{,} k\left(\sigma^{\prime}\right)\right\}_{\infty}=\left(k(\sigma) \otimes k\left(\sigma^{\prime}\right)\right) \hat{r}_{\infty}\left(a, \sigma-\sigma^{\prime}\right)-\hat{r}\left(\sigma-\sigma^{\prime}\right)\left(k(\sigma) \otimes k\left(\sigma^{\prime}\right)\right),
\end{aligned}
$$


where $a$ parametrizes the Weyl chamber $A$ in Lie $(T)$ and

$$
\hat{r}_{\infty}\left(a, \sigma-\sigma^{\prime}\right)=\sum_{\alpha} \frac{i|\alpha|^{2}}{2} \operatorname{coth}(\alpha(a)) E^{-\alpha} \otimes E^{\alpha}+\mathcal{C} \operatorname{cotg} \frac{\sigma-\sigma^{\prime}}{2} .
$$

There exists a Riemann-Hilbert map $R H: L G^{\mathbf{C}} \rightarrow B$ that is characterized by the property $l^{-1} R H(l) \in \bar{B}$ for a $l \in L G^{\mathbf{C}}$. It turns out that the limiting Poisson brackets (4) do not define an everywhere non-degenerate Poisson bivector $\Pi_{\infty}$ on the manifold $L G \times A$. However, the domain of definition of the map $R H\left(k(\sigma) e^{a}\right)$ is a dense open submanifold of $L G \times A$ on which $\Pi_{\infty}$ is non-degenerate. We denote this domain of definition as $P_{\infty}$. The Hamiltonian $H_{\infty}$ is the $\varepsilon \rightarrow \infty$ limit of $H_{\varepsilon}$.

The moment map $\mu_{\infty}: P_{\infty} \rightarrow B$ that realizes the Poisson-Lie symmetry of the $\varepsilon \rightarrow \infty$ limit of the $\varepsilon$-deformed WZW model $\left(P_{\infty}, \Pi_{\infty}, H_{\infty}\right)$ is then given by

$$
\mu_{\infty}(k(\sigma), a)=R H\left(k(\sigma) e^{a}\right) .
$$

It can be shown that $H_{\infty}$ fulfils the condition 2) of the definition of the Poisson-Lie symmetry in Section 2. Actually, the time evolution determined by the Hamiltonian $H_{\infty}$ is given by a very simple formula

$$
k(\sigma) \rightarrow k(\sigma-\tau), \quad a \rightarrow a .
$$

\section{Duality}

From the general theory of Poisson-Lie symmetry presented in Section 2, we know that both symmetry Lie algebras $\mathcal{G}_{L}=\operatorname{Lie}(L G)$ and $\mathcal{G}_{R}=\operatorname{Lie}(\bar{B})$ act on the phase space $P_{\infty}$ of the $\varepsilon \rightarrow \infty$ limit of the $\varepsilon$-deformed WZW model. Indeed, to any $\lambda \in \mathcal{G}_{L}$ it corresponds the vector field $\Pi_{\infty}\left(\cdot, \mu_{\infty}^{*} \lambda\right)$ and to any $\rho \in \mathcal{G}_{R}$ it corresponds $\Pi_{\infty}\left(\cdot, \mu_{\infty}^{*} \rho\right)$. A calculation shows that the $\mathcal{G}_{L}$ action is just the infinitesimal version of the action of $L G$ on itself:

$$
h(\sigma) \triangleright k(\sigma)=h(\sigma) k(\sigma), \quad h(\sigma), k(\sigma) \in L G,
$$

but the formula for the action of $\mathcal{G}_{R}$ turns out to be cumbersome and not very illuminating. To make this formula more friendly and transparent, we shall parametrize the phase space $P_{\infty}$ in terms of the group elements $\tilde{k}(\sigma) \in \bar{B}$ in such a way the $\mathcal{G}_{R}$ action will be just the infinitesimal version of the action of $\bar{B}$ on itself:

$$
\tilde{h}(\sigma) \triangleright \tilde{k}(\sigma)=\tilde{h}(\sigma) \tilde{k}(\sigma), \quad \tilde{h}(\sigma), \tilde{k}(\sigma) \in \bar{B} .
$$

It turns out that the following simple "coordinate" transformation on $P_{\infty}$ does the job:

$$
\tilde{k}^{-1}(\sigma)=e^{-a} k(\sigma)^{-1} R H\left(k(\sigma) e^{a}\right), \quad \tilde{a}=-a .
$$

This relation between the old description of the phase space $P_{\infty}$ in terms of $(a, k(\sigma) \in L G)$ and the new description in terms of $(\tilde{a}, \tilde{k}(\sigma) \in \bar{B})$ entails more advantages than just the elegant descriptions (5) and (6) of the both $\mathcal{G}_{L}$ and $\mathcal{G}_{R}$ symmetry actions on the phase space. In fact, it permits also to characterize $P_{\infty}$ not just as the domain of definition of the Riemann-Hilbert map but directly as $P_{\infty}=\bar{B} \times A$, which means that the infinitesimal Lie $(\bar{B})$ action can be lifted to the global $\bar{B}$ action (6).

The fundamental exchange relations, that characterize the Poisson algebra Fun $\left(P_{\infty}\right)$, can be written either in terms of the variables $(a, k(\sigma) \in L G)$ or in terms of the dual variables $(\tilde{a}, \tilde{k}(\sigma)) \in \bar{B})$ :

$$
\begin{aligned}
& \left\{k(\sigma) \stackrel{\otimes}{,} k\left(\sigma^{\prime}\right)\right\}_{\infty}=\left(k(\sigma) \otimes k\left(\sigma^{\prime}\right)\right) \hat{r}_{\infty}\left(a, \sigma-\sigma^{\prime}\right)-\hat{r}\left(\sigma-\sigma^{\prime}\right)\left(k(\sigma) \otimes k\left(\sigma^{\prime}\right)\right), \\
& -\left\{\tilde{K}(\sigma) \stackrel{\otimes}{,} \tilde{K}\left(\sigma^{\prime}\right)\right\}_{\infty}=\left(\tilde{K}(\sigma) \otimes \tilde{K}\left(\sigma^{\prime}\right)\right) \hat{r}_{\infty}\left(-\tilde{a}, \sigma-\sigma^{\prime}\right)-\hat{r}\left(\sigma-\sigma^{\prime}\right)\left(\tilde{K}(\sigma) \otimes \tilde{K}\left(\sigma^{\prime}\right)\right) .
\end{aligned}
$$


Here $\tilde{K}(\sigma) \equiv \tilde{k}(\sigma) e^{\tilde{a}}$. The resemblance of the formulae (4) and (8) is remarkable, since it is just the existence of the dual description of $P_{\infty}$ which follows from the general theory of PoissonLie symmetry, but not the invariance of the form of the exchange relations under the duality transformation (7). We interpret this form invariance as the $\mathcal{G}_{L} \leftrightarrow \mathcal{G}_{R}$ duality of the chiral $\varepsilon \rightarrow \infty$ WZW model.

\section{Conclusions}

The $\varepsilon \rightarrow \infty$ limit of the $\varepsilon$-deformed WZW model is a dynamical system in which we have discovered the Poisson-Lie symmetry with two non-isomorphic symmetry groups $G_{L}=L G$ and $G_{R}=\bar{B}$. In particular, the appearance of the group $\bar{B}$ in the story is interesting because for the finite $\varepsilon$ WZW model (including the standard case $\varepsilon=0$ ) it holds $G_{L}=G_{R}=L G$. Thus, in a sense, we may say that the symmetry pattern of the $\varepsilon \rightarrow \infty$ WZW model is richer and more intriguing than that of its finite $\varepsilon$ counterpart. The presence of the new symmetry group $\bar{B}$ turned out to be very useful for the description of the phase space of the limiting model but also for uncovering the interesting duality of the exchange relations (4) and (8). We believe that this duality is one of two circumstances which should facilitate the program of quantizing the model. The other one is the remarkable fact, that in the $\varepsilon \rightarrow \infty$ limit the elliptic exchange relations (2) simplify to the trigonometric relations (4).

\section{References}

[1] Chu M., Goddard P., Halliday I., Olive D., Schwimmer A., Quantization of the Wess-Zumino-Witten model on a circle, Phys. Lett. B 266 (1991), 71-81.

[2] Dazord P., Sondaz D., Groupes de Poisson affines, in Symplectic Geometry, Groupoids, and Integrable Systems (1989, Berkeley, CA), Editors P. Dazord and A. Weinstein, Math. Sci. Res. Inst. Publ., Vol. 20, Springer, New York, 1991, 99-128.

[3] Dorfman I.Ya., Deformations of Hamiltonian structures and integrable systems, Proceedings of Second International Workshop on Nonlinear and Turbulent Processes in Physics (October 19-25, 1983, Kyiv), Editor R.Z. Sagdeev, Harwood Academic Publishers, 1984, Vol. 3, 1313-1318.

[4] Felder G., Conformal field theory and integrable systems associated to elliptic curves, in Proceedings of the International Congress of Mathematicians (1994, Zürich), Birkhäuser, Basel, 1995, 1247-1255, hep-th/9407154.

[5] Klimčík C., Quasitriangular WZW model, Rev. Math. Phys. 16 (2004), 679-808, hep-th/0103118.

[6] Klimčík C., $q \rightarrow \infty$ limit of the quasitriangular WZW model, J. Nonlinear Math. Phys. 14 (2007), 486-518, math-ph/0611066.

[7] Koszul J.L., Crochet de Schouten-Nijenhuis et cohomologie, Astérisque 137 (1985), 257-271.

[8] Lu J.-H., Multiplicative and affine Poisson structures on Lie groups, Ph.D. Thesis, University of California, Berkeley, 1990, available at http://hkumath.hku.hk/ jhlu/publications.html.

[9] Lukyanov S., Shatashvili S., Free field representation for the classical limit of quantum affine algebra, Phys. Lett. B 298 (1993), 111-115, hep-th/9209130.

[10] Magri F., Morosi C., A geometrical characterization of integrable Hamiltonian systems through the theory of Poisson-Nijenhuis manifolds, Quaderno, Vol. 19, University of Milano, 1984.

[11] Reshetikhin N.Yu., Semenov-Tian-Shansky M.A., Central extensions of quantum current groups, Lett. Math. Phys. 19 (1990), 133-142. 